\title{
3D Modeling and Visual Simulation of Plain Knitted Structures
}

\author{
http://dx.doi.org/10.3991/ijoe.v9i5.2930 \\ Yinglin $\mathrm{Li}^{1}$, Lianhe Yang ${ }^{1}$, Suying Chen ${ }^{2}$, Jingjing Tao ${ }^{1}$ \\ ${ }^{1}$ Tianjin Polytechnic University, Tianjin, China \\ ${ }^{2}$ Qingdao University, Qingdao, China
}

\begin{abstract}
Weft knitted fabrics are widely used in clothing textiles and technical applications,such as sweater and underwear,etc. The geometrical modeling of weft knitted structures is useful to predict fabrics properties and for creating physical models. How to realize the realistic computer simulation of plain knitted structures, much research is still needed. By measuring the loop structure size of fabrics produced by the circular machine and flat machine, a 3D geometrical model of plain loop with combination of empirical and theoretical was developed. The loop model has a total of 17 data points. Cubic NURBS curve was used to generate central path of yarn in plain loop. The circle was selected as the cross-section of yarn. In Visual $\mathrm{C}++$ and OpenGL environment, then the 3D computer simulation of plain knitted fabric was realized. The simulation results distinctly show the intermeshed loops effects of knitted structures in 3D space..
\end{abstract}

Index Terms-Computer application; loop model; NURBS; plain knitted structures; 3D simulation.

\section{INTRODUCTION}

Weft knitted fabric simulation using computer graphics technology, combined with textile technology, makes designers can quickly see the intuitive fabric simulated pictures on the computer screen. It replace the process of products repeatedly try woven and proofing. This technology allows fabric simulation technology to reduce the intensity of the designers, improve work efficiency, what's more important is to shorten the development cycle of textile products, thus faster response to market changes and demand, enhance the competitiveness of enterprises.

Many geometrical models have been created by previous researchers for plain knitted fabric, including those of Chamberlain, Peirce, Leaf and Glaskin, Leaf, Munden, Postle, Demiroz and Kurbak [1]. The more commonly used is the well-known 2D Peirce model of fabric structure which put forward in the 1930s[2]. Kurbak's model is a better reflection of the intermeshed loops relationship in space[1]. However, there is a great difference between the existing theoretical plain loop model and the actual loop shape in the fabrics.

Based on experimental work, a 3D geometrical plain loop will be created.

\section{GEOMETRICAL MODELING OF PLAIN LOOP}

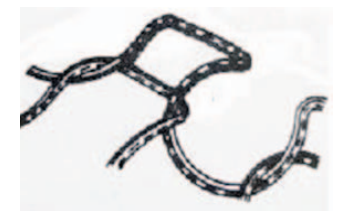

Figure 1. The shape of plain loop

Knitted structures are progressively built-up from row of intermeshed loops. The basic constitutional unit of knitted fabrics is the loop. It is a space curve bending. The geometrical shape of the plain loop is shown in Figure .1. The loop model should be developed before the simulation of the knitted fabric, and simplified as much as possible based on can reflect the basic structure of knitted fabric.

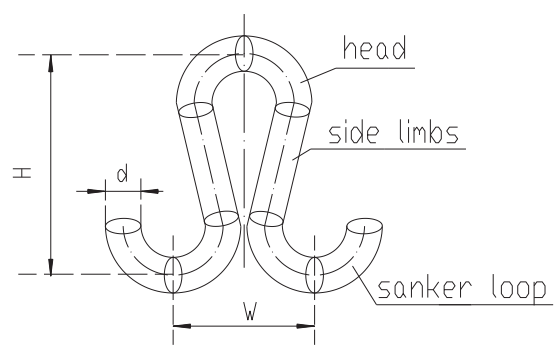

Figure 2. The structure of plain loop

The plain loop is composed of needle loop, sinker loop and loop column(as shown in Figure 2). It was assumed that the yarn in the loop is uniform when the fabric is fully relaxed, the section is circular and the loop is evenly symmetrical.

\section{A. Central path of yarn in plain loop}

The basic parameters of 3D plain loop model[3-5] are: the loop width $W$, the loop height $H$, the yarn diameter $d$, the thickness $T$ of the fabric, and the course spacing $C$ (as shown in Figure 3).

By measuring a variety of loop structure size of actual fabrics produced by the circular machine and flat machine, then calculate the average value of size, and the data points(which loop pass through) $X, Y$ coordinates on the loop based on proportional relationship with $W$ and $H$. 3D loop model still needs to consider the ups and downs 
and intermesh of loop in the thickness direction, due to inconvenience measurement, $Z$ coordinates of data points are represented by a sine function.

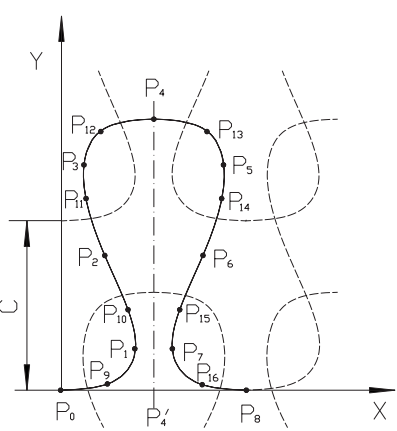

(a)Front view

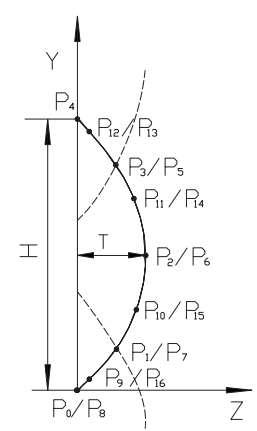

(b)Side view
Figure 3. The geometrical model of plain loop(central path)

TABLE I.

DATA POINTS COORDINATE OF PLAIN LOOP

\begin{tabular}{|c|c|c|c|}
\hline $\begin{array}{c}\text { Data } \\
\text { Points } \\
\text { No. }\end{array}$ & $\begin{array}{c}X \\
\text { Coordinate }\end{array}$ & $\begin{array}{c}Y \\
\text { Coordinate }\end{array}$ & $\begin{array}{c}Z \\
\text { Coordinate }\end{array}$ \\
\hline$P_{0}$ & 0 & 0 & 0 \\
\hline$P_{1}$ & $0.3493 \mathrm{~W}$ & $0.2035 H$ & $T \sin (0.2035 \pi)$ \\
\hline$P_{2}$ & $0.2398 \mathrm{~W}$ & $0.4954 H$ & $\mathrm{~T} \sin (0.4954 \pi)$ \\
\hline$P_{3}$ & $0.1488 \mathrm{~W}$ & $0.7874 H$ & $T \sin (0.7874 \pi)$ \\
\hline$P_{4}$ & $0.5000 \mathrm{~W}$ & $H$ & 0 \\
\hline$P_{5}$ & $0.8512 \mathrm{~W}$ & $0.7874 H$ & $T \sin (0.7874 \pi)$ \\
\hline$P_{6}$ & $0.7602 \mathrm{~W}$ & $0.4954 H$ & $T \sin (4954 \pi)$ \\
\hline$P_{7}$ & $0.6507 \mathrm{~W}$ & $0.2035 H$ & $T \sin (0.2035 \pi)$ \\
\hline$P_{8}$ & $W$ & 0 & 0 \\
\hline$P_{9}$ & $0.2500 \mathrm{~W}$ & $0.0407 H$ & $T \sin (0.0407 \pi)$ \\
\hline$P_{10}$ & $0.3145 \mathrm{~W}$ & $0.3494 \mathrm{H}$ & $T \sin (0.3494 \pi)$ \\
\hline$P_{11}$ & $0.1751 \mathrm{~W}$ & $0.6486 \mathrm{H}$ & $\mathrm{T} \sin (0.6486 \pi)$ \\
\hline$P_{12}$ & $0.2190 \mathrm{~W}$ & $0.9230 \mathrm{H}$ & $\mathrm{T} \sin (0.9230 \pi)$ \\
\hline$P_{13}$ & $0.7810 \mathrm{~W}$ & $0.9230 \mathrm{H}$ & $\mathrm{T} \sin (0.9230 \pi)$ \\
\hline$P_{14}$ & $0.8249 \mathrm{~W}$ & $0.6486 H$ & $\mathrm{~T} \sin (0.6486 \pi)$ \\
\hline$P_{15}$ & $0.6855 \mathrm{~W}$ & $0.3494 \mathrm{H}$ & $\mathrm{T} \sin (0.3494 \pi)$ \\
\hline$P_{16}$ & $0.7500 \mathrm{~W}$ & $0.0407 H$ & $T \sin (0.0407 \pi)$ \\
\hline
\end{tabular}

Data points coordinate of plain loop are presented by proportional relationship of loop parameters(as shown in Table I).

The central path of the loop model is irregular space curve which passes through the data points (as shown in Figure 3 ), it requires of the curve is $C^{2}$ continuous when the connection of segments. Because NURBS curve can be partially adjusted, and has good geometry characteristic and high flexibility, it can simulate the trend of the centerline of the yarn well, so using cubic NURBS curve to simulate yarn path[6-8]. The central path of the loop model is generated by NURBS curve of passing through the data points.

The formula of cubic NURBS curve[6-8] is:.

$$
p(u)=\frac{\sum_{i=0}^{n} w_{i} d_{i} N_{i, 3}(u)}{\sum_{i=0}^{n} w_{i} N_{i, 3}(u)}, i=0,1,2, \ldots, n
$$

where $w_{i}$ is the weight factor, $d_{i}$ is the control point, and $N_{i, 3}(u)$ is the 3 degree B-Spline basic function.

According to NURBS method, the control points of curve can be inversely calculated by the given data points, and then the shape of the curve can be derived by interpolation.

\section{B. Cross-section of yarn in plain loop}

There are many kinds of cross-section model, like circle, ellipse, runway, convex lens, etc. Because that the equation of circular cross-section is simple, and its parameter is few, only decided by diameter $d$, which easier to achieve parameterization. Therefore circular was selected as cross-section of yarn in plain loop model.

\section{SIMULATIUON ALGORITHM DESIGN}

Plain knitted fabric is consisting of the $\mathrm{m}$ pattern rows, $\mathrm{n}$ wales loop unit, its simulation algorithm $[9,10]$ is as follows:

1) Enter the loop parameters: the loop width $W$, the loop height $H$, the yarn diameter $d$, the course spacing $C$ and fabric thickness $\mathrm{T}$.

2) Enter the loop pattern row number $m$ and wale number $n$.

3) Enter the start point's coordinates of the first loop.

4) According to the loop parameters and start point's coordinate, as well as the relationship between the data points and loop parameters in Table I, calculate every data point's coordinates of the first loop.

5) Using the data points of loop to calculate inversely control points, making NURBS curve passing through these data points, calculate every interpolation point's coordinates of the first loop, then save them in the Object of class CObArray of MFC.

6) The coordinates of each point in pattern row number $i$, wale number $j$ are come from each interpolation point's coordinates in the first loop. The coordinates by making $w^{*}(j-1)$ offset in x-coordinate and $C^{*}(i-1)$ offset in $\mathrm{y}-$ coordinate can be obtained. Then save all points' coordinates in the template array.

7) Using $d$ as diameter, each interpolation point's coordinates of the loop as the center of sphere, draw small spheres with the function auxSolidSphere() of OpenGL.

For the reason that the interpolation points of the loop are close enough, the small spheres along the yarn central path are plenty. So the yarn effect is expressed.

\section{SimAlation RESUlts}

In Visual $\mathrm{C}++$ and OpenGL platform, 3D visual simulation program was written according to the simulatiuon algorithm mentioned above.

Figure 4 shown the central path of the plain loop model. Figure 5 is the $3 \mathrm{D}$ simulation effect of weft knitted fabric. Figure 6 is photomicrograph of the real plain structures. Compared with real fabric and simulation, simulation results distinctly shows the intermeshed loops of knitted structure in $3 \mathrm{D}$ space. 
PAPER

3D Modeling and Visual Simulation of Plain KNITTED StRUCtures

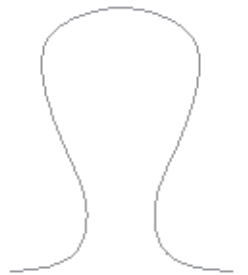

(a) Front view

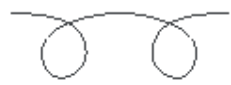

(c) Top view

Figure 4. The central path of plain loop

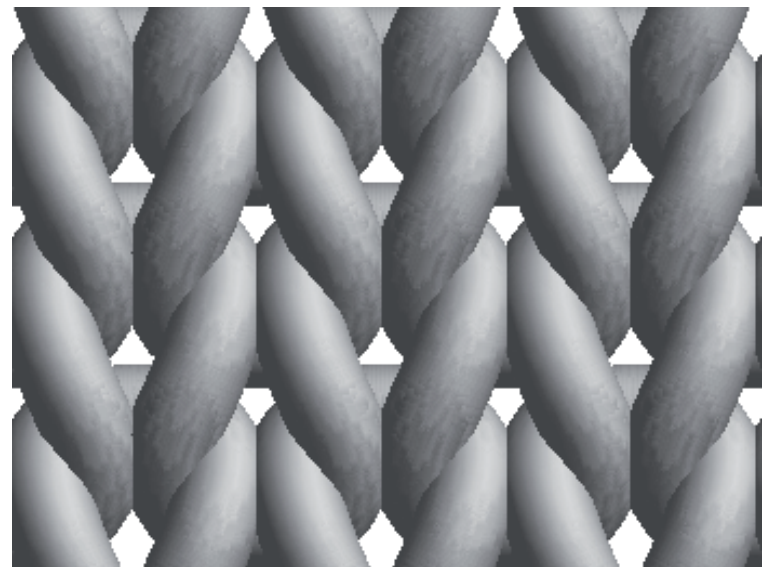

Figure 5. The simulation of plain structures

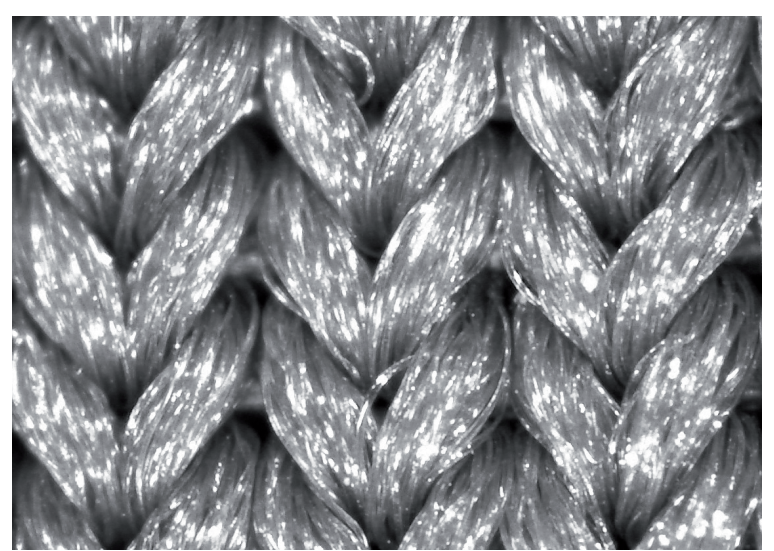

Figure 6. Photomicrograph of the real plain structures

\section{CONCLUSIONS}

In basis of experimental work, a 3D geometrical plain loop model combinating of empirical and theoretical was developed. Using cubic NURBS curve generates the central path of yarn. 3D simulation was created by sweeping the central path with circle along the yarn. It can be successful used to create basic weft knitted structures on the computer. In Visual C++ and OpenGL environment, 3D computer simulation of plain knitted fabric has been realized. The simulation results is excellent accuracy and realism.
The graphics using 3D loop model can reflect the characteristics of knitted fabrics better, different colors and diameter of the loop generated different styles of knitting rendering.

Because of the complexity of the structure and the diversity of the material, 3D simulation of knitted fabric still needs long-term and plenty research to achieve more vivid effect.

\section{REFERENCES}

[1] Arif Kurbak, Ozgur Ermen, "Basic Studies for Modeling Complex Weft Knitted Fabric Structures Part I: A Geometrical Model for Widthwise Curlings of Plain Knitted Fabrics,".Textile Research Journal. London, vol. A78 , pp. 198-208, April 2008. http://dx.doi.org/10.1177/0040517507082352

[2] Liu Su, Long Hai-ru, "Three-dimensional computer simulation of plain weft knitted fabric". Journal of Textile Research. Beijing. vol. A28, pp.41-44, December 2007.

[3] Arif Kuabak, Tuba Alpyildiz. A Geometrical Model for the Double Lacoste Knits. Textile Research Journal. London, vol. A78, pp. 232-247, March 2008.

[4] Arif Kurbak, Ozlem Kayacan, "Basic Studies for Modeling Complex Weft Knitted Fabric Structures Part II: A Geometrical Model for Plain Knitted Fabric Spirality". Textile Research Journal. London, vol. A78, pp. 279-278, April 2008. http://dx.doi.org/10.1177/0040517507082351

[5] LI Yinglin, YANG Lianhe, CHEN,Suying, "3D Modeling and Simulation of Fancy Fabrics in Weft Knitting", Journal of Donghua University (Eng. Ed.). Shanghai, vol. A29, pp. 351-358, August 2012.

[6] O.Goktepe, S.C.Harlock, "A 3D Loop Model for Visual Simulation of Warp-knitted Structures". Journal of the Textile Institute.London, vol. A93, pp. 11-28, May 2002.

[7] O.Goktepe, S.C.Harlock, "Three-Dimensional Computer Modeling of Warp Knitted Structures". Textile Research Journal. London, vol. A72, pp. 266-272, April 2002. http://dx.doi.org/10.1177/004051750207200314

[8] R.B. Turan, G. Baser. Three-Dimensional Computer simulation of $2 / 2$ twill woven fabric by using B_splines. The Journal of The Textile Institute, Vol. A101, pp. 870-881, Octobert 2010.

[9] Deng Zhong-min, Wang Li-jing, "Enhanced Visualization of Simulated Woven Fabrics". Fibers and Polymers, vol. A11, pp.531-536, March 2010.

[10] LIAO Tianyi, SABIT Adanur, "A Novel Approach to ThreeDimensional modeling of Interlaced fabric Structures", Textile Research Journal.London, Vol. A68, pp. 841-847, November 1998.

\section{AUTHORS}

Yinglin $\mathbf{L i}$ is with School of Textile, Tianjin Polytechnic University, Tianjin, 300160, China (e-mail: liyinglin@tjpu.edu.cn).

Lianhe Yang(Corresponding author) is with School of Computer Science \& Software Engineering, Tianjin Polytechnic University, Tianjin, 300160, China (e-mail: yanglh@tjpu.edu.cn).

Suying Chen is with School of Textile and Clothing,Qingdao University, Qingdao, Shandong, 266071, China (e-mail:).

Jingjing Tao is with School of Textile, Tianjin Polytechnic University, Tianjin, 300160, China (447398341@qq.com).

This work was supported by Natural Science Foundation of Tianjin, China (No.11JCYBJC26400). Submitted 20 June 2013. Published as resubmitted by the authors 15 September 2013. 